AUDIT

\title{
Neonatal murmurs: are senior house officers good enough?
}

\section{K F M Farrer, J M Rennie}

Arch Dis Child Fetal Neonatal Ed 2003;88:F147-F151

See end of article for authors' affiliations

.....................

Correspondence to: Dr Farrer, Neonatal Intensive Care Unit, St George's Hospital,

Tooting, London SW17 OQT, UK; kate.farrer@stgeorges.nhs.uk

Accepted 9 August 2002
Aim: To show that, given appropriate guidelines, senior house officers (SHOs) have the clinical skills required to assess neonatal murmurs.

Methods: Neonatal SHOs identified babies with a cardiac murmur at routine neonatal examination. The $\mathrm{SHOs}$ assessed whether the murmur was significant or innocent and decided between immediate further assessment or echocardiogram as an outpatient.

Results: A total of 112 babies had murmurs at routine neonatal examination. The incidence of cardiac murmurs was 13.8 per 1000. Twelve babies were referred for immediate further assessment. Eleven had structurally abnormal hearts. One had a normal heart with pulmonary hypoplasia. One hundred babies were referred, and 78 attended for outpatient follow up. Of these, the SHO assessed nine babies as having a significant murmur and 69 as having an innocent murmur. Twenty two babies failed to attend for follow up; all were thought to have innocent murmurs. Of the nine murmurs assessed as significant, four were confirmed as such and five were found to be innocent. Of the 91 murmurs assessed as innocent, 63 were proven to be innocent, six had abnormalities on echocardiogram, and 22 defaulted to follow up. Five of the serious murmurs were small ventricular septal defects, which had resolved by 6 months of age; the other had mild pulmonary stenosis. None of these babies were clinically symptomatic at outpatient review.

Conclusion: Given appropriate guidelines, SHOs have the skills to assess the significance of, and decide on appropriate management for, neonatal murmurs. Electrocardiograms and chest radiographs are not necessary.
$\mathrm{T}$ he management of babies found to have a cardiac murmur at the routine neonatal examination varies between neonatal units. Initial management may involve review by the registrar or consultant plus an electrocardiogram (ECG) and chest radiograph (CXR). These actions may delay discharge and cause parental anxiety.

Delay in diagnosis of congenital heart disease (CHD) can have serious consequences, causing compromise to the baby and distress to the family. A normal neonatal examination does not exclude CHD, and routine examination may fail to detect more than half of the cases of $\mathrm{CHD}{ }^{1}$ The routine neonatal examination performs poorly as a screening test. ${ }^{2}$ An echocardiogram is necessary for accurate diagnosis of CHD. However, clinical examination can determine the presence or absence of CHD and thus an echocardiogram may be unnecessary for those with clinically innocent murmurs many of which resolve spontaneously. ${ }^{3-5}$ Immediate echocardiogram is not available in most units, and early referral to a paediatric cardiology centre is suggested. ${ }^{26}$ However, delay in assessment is inevitable in some cases, thus it is highly desirable that senior house officers (SHOs) are maximally equipped with the skills to detect significant heart disease as early as possible.

Unless an SHO has taken a postgraduate examination, their clinical skills are rarely observed and assessed. Examination of the newborn is well documented in clinical texts, but SHOs may only read these if studying for postgraduate examinations, and many SHOs may only be equipped with the neonatal examination routine taught as a medical student.

The aim of our study was to show that, given appropriate guidelines:

(1) SHOs are able to assess the significance of cardiac murmurs in newborn babies;

(2) SHOs can assess the need for immediate review or outpatient follow up;

(3) it is safe to dispense with routine ECGs and CXRs.

\section{SUBJECTS AND METHODS}

This study was carried out during a two year period at a tertiary referral teaching hospital. Subjects included all babies on the postnatal wards who had a routine neonatal examination by an SHO. Excluded from this study were babies who had abnormal antenatal echocardiograms, dysmorphic babies such as those with Down's syndrome, those who needed intensive care, and those who presented as unwell before the routine examination.

The routine neonatal examination was performed before discharge, usually the day after delivery, but the examination was sometimes inevitably delayed.

The SHOs were attached to the neonatal unit for a period of six months. Their previous experience varied from a total of only six months paediatric experience to substantial experience and possession of postgraduate paediatric qualifications. At the start of their attachment, the SHOs were directed to the unit's guidelines on the management of babies found to have a cardiac murmur. These referred to the clinical signs of an innocent murmur $^{7-9}$ and then advised:

(1) Any baby with cyanosis, cardiac failure, or impalpable or decreased pulses should be admitted to the neonatal unit for further assessment (group 1).

(2) Discussion with a senior colleague about any case with a positive family history or concerning antenatal scan (group 2).

(3) Referral to next neonatal cardiac clinic if the murmur was loud ( $\geqslant$ grade $3 / 6$ ), harsh, diastolic, pansystolic, or continuous,

Abbreviations: $E C G$, electrocardiogram; CXR, chest radiograph; $C H D$, congenital heart disease; $\mathrm{SHO}$, senior house officer; VSD, ventricular septal defect; PDA, patent ductus arteriosus; "PBS", mild pulmonary branch stenosis 
Table 1 Characteristics of the 112 babies found to have heart murmurs

\begin{tabular}{ll}
\hline Males & 59 \\
Females & 53 \\
Gestation (weeks) & $39.4(35.3-42.3)$ \\
Birth weight (g) & $3380(2160-5360)$ \\
Age at neonatal check & $1.5(1-8)$ \\
(days) & \\
\hline
\end{tabular}

Values are mean (range).

or was associated with an ejection click, or praecordial hyperactivity (group 3).

(4) Non-urgent referral to neonatal cardiac clinic if the baby had characteristics of a clinically innocent murmur. These babies were feeding well, pink, and well perfused with a normal heart and respiratory rate and no sign of respiratory distress. The praecordium was quiet with normal pulses, a soft systolic murmur, and no hepatomegaly. Parents of these babies were given information about the significant symptoms and signs to look for should the baby become compromised and to seek early medical opinion in this event (group 4).

If, despite assessing a case as appropriate for outpatient follow up, the SHO preferred to have a senior opinion before discharge, then this was arranged.

Babies referred to outpatients for follow up were seen by a single consultant neonatologist (JR) who clinically assessed the baby and performed an echocardiogram. The consultant's training had consisted of attending a course and consolidating knowledge by experience. Standard views were obtained; colour and pulsed and continuous wave Doppler were available to assist in the identification of these defects. Babies found to have an abnormality on echocardiogram were either referred to the regional paediatric cardiology centre at Guy's Hospital for further assessment or had a later review by the consultant neonatologist. If the abnormality persisted, referral to Guy's was arranged. No baby had an ECG or CXR unless he or she was symptomatic.

\section{RESULTS}

During the study period, there were 8904 live births at this hospital. Of these, 808 were excluded from the study population. Thus 8096 babies had routine neonatal examinations on the postnatal wards.

A total of 112 babies were found to have a cardiac murmur on routine neonatal examination. The prevalence in this population was 13.8 per 1000 . Table 1 shows the characteristics of the babies in the study.

\section{Group 1}

Twelve babies had a murmur with worrying clinical features and thus had immediate further senior assessment (fig 1). Their diagnoses were: ventricular septal defect (VSD) (five cases), pulmonary stenosis (two cases), tetralogy of Fallot (two cases), transposition of the great arteries (one case), atrial septal defect and VSD (one case) and one case of pulmonary hypoplasia with a normal heart.

\section{Group 2}

During the study period, eight babies with normal clinical examinations, but concerning family history or antenatal scans, were referred for an outpatient echocardiogram with the consultant neonatologist. Four had siblings who had significant CHD, three babies had antenatally diagnosed left

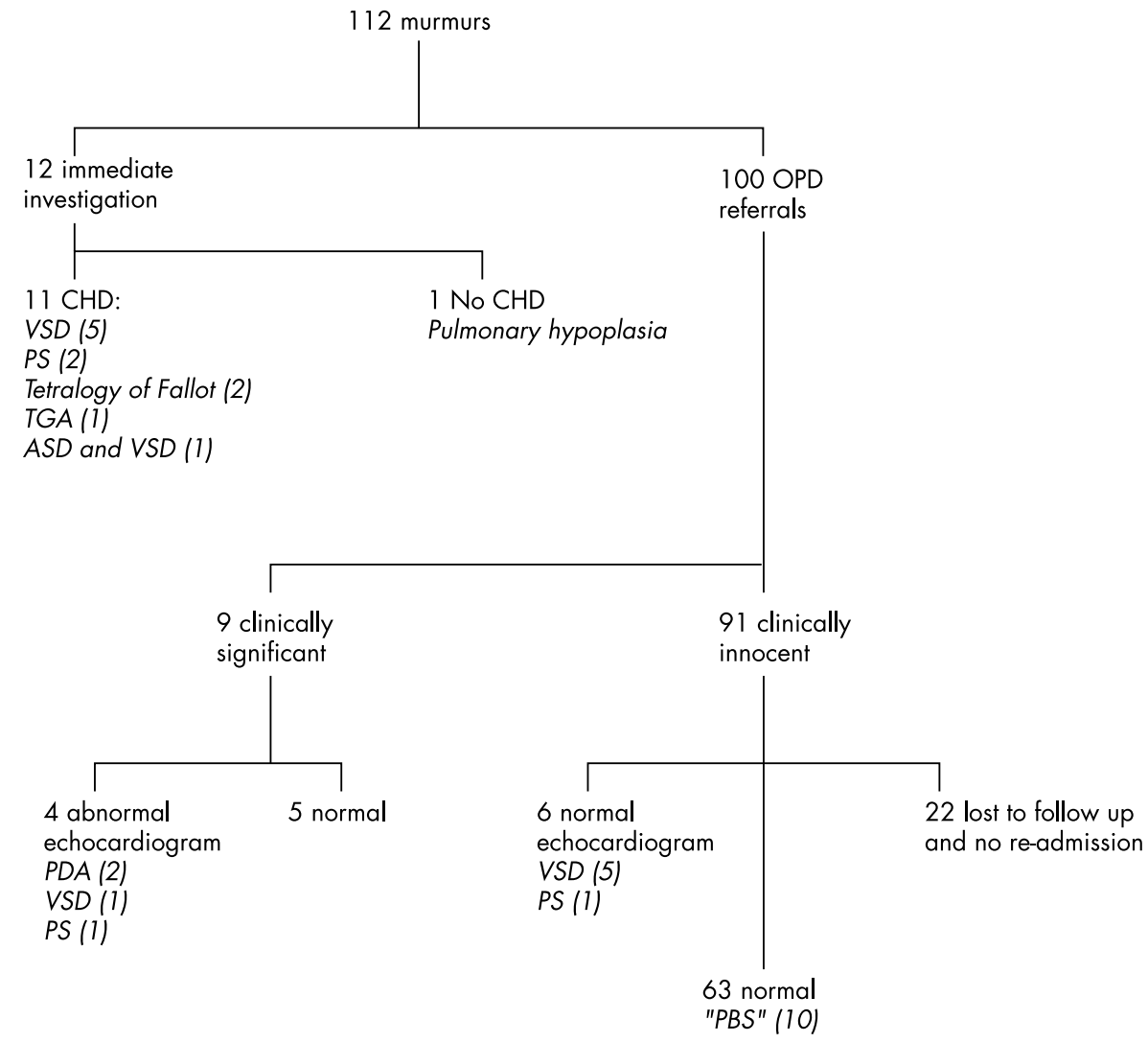

Figure 1 Echocardiogram results of babies with murmur at newborn examination. CHD, Congenital heart disease; VSD, ventricular septal defect; PS, pulmonary stenosis; TGA, ; ASD, atrial septal defect; OPD, outpatient department; PDA, patent ductus arteriosus; "PBS", mild pulmonary branch stenosis. 


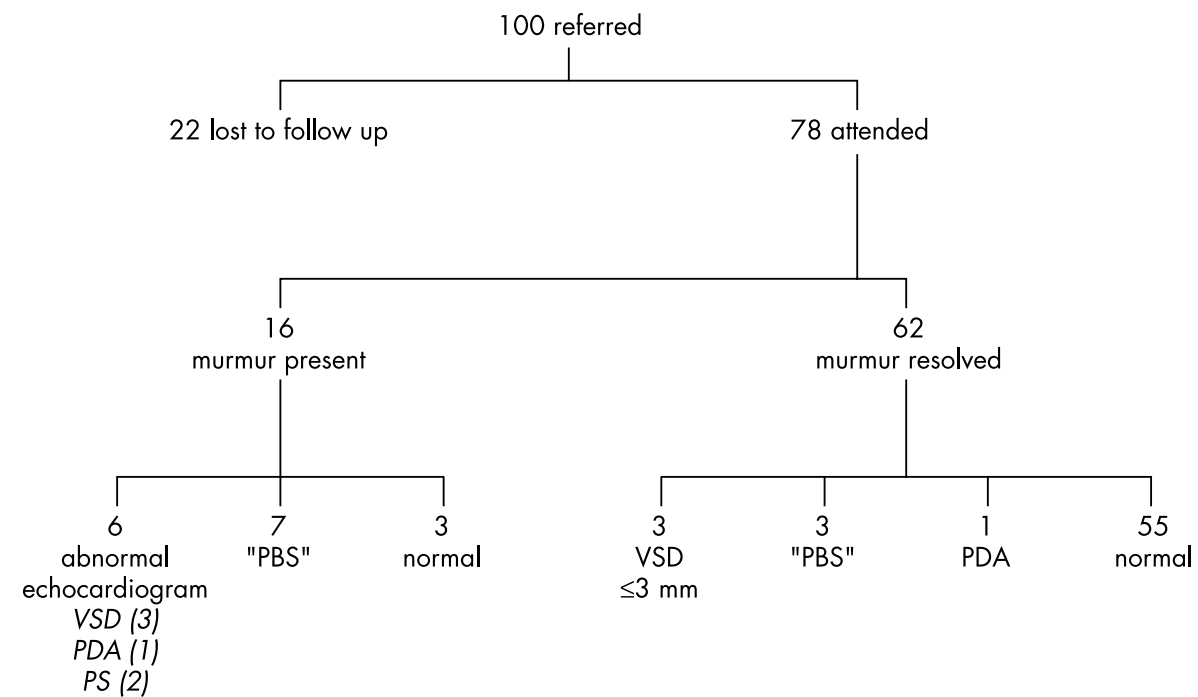

Figure 2 Echocardiogram results of babies with murmur at outpatient follow up. VSD, Ventricular septal defect; PS, pulmonary stenosis; PDA, patent ductus arteriosus; "PBS", mild pulmonary branch stenosis.

ventricular "golf-balls", and one baby had a small antenatal pleural effusion. All of them had a normal postnatal echocardiogram.

One hundred babies had a cardiac murmur on routine neonatal examination and were referred to the outpatient neonatal cardiac clinic for an echocardiogram by the consultant neonatologist (fig 1); 78 attended for follow up. The total mean waiting time for all babies was nine weeks (range four days to 15 weeks).

\section{Group 3}

Despite appearing to have a clinically significant cardiac murmur on neonatal examination, nine babies were assessed by the SHO as being clinically well enough to be referred for outpatient cardiac assessment. They all had either a hyperdynamic praecordium or harsh murmur. The mean waiting time was seven weeks (range four days to 14 weeks). Of these, five had a normal echocardiogram and four had abnormalities on the echocardiogram: patent ductus arteriosus (PDA) (two cases), small VSD (one case), and pulmonary stenosis (one case).

\section{Group 4}

Ninety one babies were assessed at routine neonatal examination as having clinically innocent murmurs; 69 attended for follow up. The mean waiting time was 10 weeks (range 3-14). Of these, 63 had structurally normal hearts. Six had abnormalities on echocardiogram: VSD (five cases) and mild pulmonary stenosis (one case). All of the VSDs had resolved by 6 months of age.

The murmur was still present at the first outpatient appointment in 16/78 (21\%) of the babies who attended for an outpatient echocardiogram from groups 3 and 4 (fig 2). Of these, six had abnormalities on echocardiogram (PDA (one), VSD (three), PS (two)), seven had mild pulmonary branch stenosis ("PBS"), and three had normal hearts. In 62/78 (79\%) of babies, the murmur had gone at outpatient review; on echocardiography, 55 had normal hearts, three had "PBS", one had a PDA, and three had VSDs $\leqslant 3 \mathrm{~mm}$, which all resolved spontaneously.

In total, 10 babies had mild "PBS". They had increased flow, or turbulence, in the main or branch pulmonary arteries, but in all cases this was an increase of less than $50 \%$ above that in the main pulmonary trunk and thus did not reach the accepted criteria for the diagnosis of PBS. ${ }^{10}$ This resolved quickly in five babies. Turbulent flow in the pulmonary artery persisted in five

\begin{tabular}{|c|c|c|c|}
\hline \multirow[b]{2}{*}{ Clinically } & \multicolumn{2}{|c|}{ Echo result } & \multirow[b]{2}{*}{ Total } \\
\hline & $\mathrm{CHD}$ & No CHD & \\
\hline $\begin{array}{l}\text { Innocent } \\
\text { Significant } \\
\text { Total }\end{array}$ & $\begin{array}{l}6 \\
15 \\
21\end{array}$ & $\begin{array}{l}63 \\
6 \\
69\end{array}$ & $\begin{array}{l}69 \\
21 \\
90\end{array}$ \\
\hline \multicolumn{4}{|c|}{$\begin{array}{l}\text { Sensitivity = true positive/all abnormal echo }= \\
15 / 21=71 \% ; \text { specificity }=\text { true negative/all } \\
\text { normal echo }=63 / 69=91 \% ; \text { positive predictive } \\
\text { value = positive test correctly identified }=15 / 21 \\
=71 \% \text {; negative predictive value = negative test } \\
\text { correctly identified }=63 / 69=91 \% ; \text { accurate } \\
\text { assessment } 78 / 90=87 \% \text {. } \\
C H D, \text { Congenital heart disease. }\end{array}$} \\
\hline
\end{tabular}

babies who were all referred to Guy's; they all had structurally normal hearts and were discharged from follow up.

Twenty two babies failed to attend for outpatient follow up. A letter was sent to the general practitioner inviting re-referral if the murmur persisted. According to the medical notes at the hospital of their birth, none of these babies subsequently presented to this hospital with cardiac problems. Furthermore, according to hospital records and the available general practitioner records, none of these babies attended the regional paediatric cardiology department at Guy's. One baby was followed up in the hepatitis B clinic, as he required a full course of hepatitis B immunisation. The murmur did not persist.

\section{Senior review}

A senior colleague assessed all the cases that had worrying clinical features. Of the cases referred to outpatients, review by the registrar was sought in 24/100 cases by $8 / 30$ SHOs. In 23 of these cases, the registrar agreed with the SHO's assessment. In one case, the SHO had assessed a murmur as significant, and the registrar correctly changed the diagnosis to innocent. Interestingly, in none of the six cases assessed by the SHO as innocent, but which subsequently were shown to have abnormalities on echocardiogram, did the SHO ask for a senior review.

All babies who attended for outpatient follow up were asymptomatic at review. Five of the babies thought to have clinically significant murmurs were found to have normal 
hearts, and six of those thought to have clinically innocent murmurs had abnormalities on echocardiogram (table 2). The sensitivity of the SHO examination to assess the clinical significance of the neonatal murmur was $71 \%$, and the specificity was $91 \%$; the positive predictive value was $71 \%$, and the negative predictive value was $91 \%$.

\section{DISCUSSION}

Previous studies have shown that $0.6-60 \%$ of neonates have a murmur at some stage, and more recent studies have shown that $0.6-1.6 \%$ of babies have a murmur at the routine neonatal examination. $^{3511-13}$ Prevalence varies according to size of study, the study population, the age at echocardiogram, the experience of the clinical examiner, and whether the study is retrospective or prospective. Some $18-86 \%$ of babies with a murmur may have CHD when assessed by echocardiography very early in life. ${ }^{3512}$ In our study, $1.38 \%$ of babies had a murmur at the routine neonatal examination. Abnormalities were identified in $21 / 90(23 \%)$ of the cohort who had an echocardiogram. Given that resources are scarce, and the natural history of innocent murmurs is resolution, it may be appropriate to delay further assessment of clinically innocent or asymptomatic murmurs where immediate echocardiogram assessment is not readily available. The constraints of the system in our hospital meant that outpatient follow up was unfortunately delayed to a mean of nine weeks (range 4 days to 15 weeks). The ideal time for further assessment is unclear. Most babies who die from CHD in the neonatal period become symptomatic before 18 hours of age. ${ }^{14}$ Almost three quarters $(70 \%)$ of the cases of CHD in a large study by Abu-Harb et al ${ }^{2}$ would have presented by 6 weeks of age. Wren et al ${ }^{1}$ showed that, if every baby who had clinical markers of CHD were examined within 4 weeks of the abnormalities being noted, then $58 \%$ could have been diagnosed by 6 weeks and $76 \%$ by 3 months of age. Most (64\%) innocent murmurs resolve by 6 weeks of age. ${ }^{5}$ Thus $4-6$ weeks of age may be the optimum time for echocardiographic assessment. However, none of our babies was unwell before, or at, the follow up appointment, thus, if the babies are selected appropriately, later assessment may be acceptable.

In our study group, there were no cases of obstruction of the left outflow tract. This may be fortuitous, or explained by the fact that the study was too small, or that these babies presented as unwell before or after the routine examination. Unfortunately the neonatal and 6 week examinations perform poorly as screening tests for diagnosis of obstructive left heart malformations in infancy. ${ }^{2}$ It is these conditions that emphasise the need for parental education on significant signs and symptoms before discharge of a baby with a murmur awaiting an echocardiogram.

The SHOs accurately assessed the significance of the murmur in $87 \%$ of babies. Previous studies have assessed the diagnostic accuracy of paediatricians' clinical auscultation skills to assess murmurs in children and cardiac simulators. $^{3}{ }^{15-17}$ None specifically looked at the clinical accuracy of SHOs (or residents) in assessing murmurs at the newborn check. The study of Du et al in term and preterm neonates showed that $90 \%$ of heart diseases were accurately diagnosed clinically. These babies were initially seen by residents, but then reviewed by a senior paediatrician, and it is not clear whether the senior paediatrician changed any of the residents' diagnoses. Hansen et al ${ }^{15}$ concluded that general paediatricians were good at diagnosing innocent murmurs, but Haney et $\mathrm{al}^{16}$ concluded that the diagnostic accuracy of office based (general) paediatricians was suboptimal. McCrindle et $_{\text {al }}{ }^{17}$ concluded that paediatric cardiologists have excellent diagnostic accuracy, but in the study of Gaskin et al, ${ }^{18}$ paediatric residents had only 33\% diagnostic accuracy.

The SHOs found the guidelines a useful framework and reference. Despite this, on 24 occasions the SHO still sought sen- ior opinion even though they had assessed a murmur as suitable for delayed outpatient review. This action was not discouraged, as we were aware that the confidence, experience, and skills of SHOs varies. In all but one case the senior doctor agreed with the SHO's assessment; the senior doctor correctly changed the SHO's assessment of significant murmur to innocent.

Many units are dispensing with the use of ECG and CXR for assessment of murmurs found at the routine neonatal examination. None of the clinically well babies in this study had an ECG or CXR as an aid to diagnosis. In no case did we feel that an ECG or CXR would have altered the outcome. Non-invasive tests as an aid to diagnosis have not been evaluated in large neonatal studies. However, evaluation in the older paediatric population with heart murmurs shows that these investigations do not alter the clinical assessment; ECG and CXR may only assist in diagnosis in those who clinically have CHD and would thus need definitive echocardiogram diagnosis. ${ }^{19-22}$

Structural abnormalities were identified in $21(23 \%)$ of the cohort who had had an echocardiogram. Eleven of these were clinically symptomatic at the routine neonatal examination and thus had immediate further investigations (one baby was correctly referred for immediate assessment in group 1 and subsequently found to have pulmonary hypoplasia with a normal echocardiogram). Ten babies had echocardiogram abnormalities when seen in the neonatal cardiac clinic (fig l). The regional paediatric cardiology team kept one baby with pulmonary stenosis under review; all other babies had a normal echocardiogram by 6 months of age. Other studies have shown up to 9 per 1000 infants have CHD presenting in infancy. ${ }^{32}{ }^{13}$ Thus up to 70 infants would be predicted to have CHD from the total 8904 babies born during the study period. This study assessed SHO's examination skills at the routine neonatal examination; 21 cases of CHD were identified. Close analysis of the number of babies who were excluded from the cohort because of antenatal diagnosis of CHD or because they presented before, or after, the neonatal examination was not the aim of the study. Thus we are unable to give an accurate number of all babies who were born during the study period who had CHD; we recognise this as a limitation of our study, but full analysis of this problem was not an aim of the study.

Half of the whole cohort with a murmur (11/22) had a VSD. Of these, six were diagnosed in the outpatients department. Five of them were babies thought by the SHO to have had clinically innocent murmurs; all these VSDs resolved by 6 months of age. VSDs are thought classically not to be associated with an early murmur, thus it is impossible to know if the murmur heard was due to the VSD or a coincidental finding.

Our study supports the view that, given appropriate guidelines, SHOs can assess the clinical significance of neonatal murmurs and decide on appropriate immediate or delayed follow up. ECG and CXR are not necessary.

\section{ACKNOWLEDGEMENTS}

We thank the staff of Guy's Hospital Paediatric Cardiology Department for their help and cooperation. We are particularly grateful to Ms Alexandra Savis, paediatric echocardiographer, for help with data collection.

\section{Authors' affiliations}

K Farrer, Neonatal Intensive Care Unit, St George's Hospital, Tooting, London SW 17 OQT, UK

J Rennie, Neonatal Intensive Care Unit, Kings College Hospital,

Denmark Hill, London SE5 9RS, UK

\section{REFERENCES}

1 Wren C, Richmond S, Donaldson L. Presentation of congenital heart disease in infancy: implications for routine examination. Arch Dis Child Fetal Neonatal Ed 1999;80:F49-53. 
2 Abu-Harb M, Wyllie J, Hey E, et al. Presentation of obstructive left heart malformations in infancy. Arch Dis Child Fetal Neonatal Ed 1994;71:F179-83

3 Du Z-D, Roguin N, Barak M. Clinical and echocardiographic evaluation of neonates with heart murmurs. Acta Paediatr 1997:86:752-6.

4 Geva T, Hegesh J, Frand M. Reappraisal of the approach to the child with heart murmurs: is echocardiography mandatory? Int J Cardiol 1988; 19:107-13.

5 Arlettaz R, Archer N, Wilkinson AR. Natural history of innocent heart murmurs in newborn babies: controlled echocardiographic study. Arch Dis Child Fetal Neonatal Ed 1998:78:F166-70.

6 Hall DMB. Health for all children. Report of the Third Joint Working Party on CHS. Oxford: OUP, 1996.

7 Field DJ, Stroobant J, Carter E, et al. Paediatrics: an illustrated colour text. Edinburgh, Churchill Livingstone, 1997:54.

8 Silove E. Assessment and management of congenital heart disease in the newborn by the district paediatrician. Arch Dis Child Fetal Neonatal Ed 1994;70:F71-4.

9 American Heart Association. Guidelines for evaluation and management of common congenital cardiac problems in infants, children and adolescents. Circulation 1994:90:2180-8

10 Arlettaz R, Archer N, Wilkinson AR. Closure of the ductus arteriosus and development of pulmonary branch stenosis in babies of less than 32 weeks gestation. Arch Dis Child Fetal Neonatal Ed 2001;85:F197-200

11 Braudo M, Rowe RD. Auscultation of the heart: early neonatal period. American Journal of Diseases in Children 1961:101:575-86.

12 Ainsworth SB, Wyllie JP, Wren C. Prevalence and clinical significance of cardiac murmurs in neonates. Arch Dis Child Fetal Neonatal Ed $1999 \cdot 80 \cdot F 43-5$
13 Rein AJ, Omokhodion SI, Nir A. Significance of cardiac murmur as the sole clinical sign in the newborn. Clin Pediatr (Phila) 2000;39:511-20.

14 Beebe SA, Britton JR, Britton HL, et al. Neonatal mortality and length of newborn hospital stay. Pediatrics 1996;98:231-5.

15 Hansen LK, Birkebaek NH, Oxhoj H. Initial evaluation of children with heart murmurs by the non-specialised paediatrician. Eur J Pediatr 1995; 154:15-17.

16 Haney I, Ipp M, Feldmen W, et al. Accuracy of clinical assessment of heart murmurs by office-based (general practice) paediatricians. Arch Dis Child 1999:81:409-12.

17 McCrindle BW, Shaffer KM, Kan JS, et al. Cardinal clinical signs in the differentiation of heart murmurs in children. Arch Pediatr Adolesc Med 1996; 150:169-74

18 Gaskin PRA, Owens SE, Talner NS, et al. Clinical auscultation skills in pediatric residents. Pediatrics 2000;105: 1184-7.

19 Smythe JF, Teixeira OHP, Vlad P, et al. Initial evaluation of heart murmurs: are laboratory tests necessary? Pediatrics 1990;86:497-500.

20 Birkebaek NH, Hansen LK, Oxhoj H. Diagnostic value of chest radiography and electrocardiography in the evaluation of children with a clinical murmur. Acta Paediatr 1995:84:1379-81.

21 Newburger J, Rosenthal A, Williams R, et al. Non-invasive tests in the initial evaluation of heart murmurs in children. N Engl J Med 1983:308:61-4.

22 Castello-Herbreteau B, Vaillant MC, Magontier N, et al. Diagnostic value of physical examination and electrocardiogram in the initial evaluation of heart murmurs in children. Arch Pediatr 2000;7:1041-9.

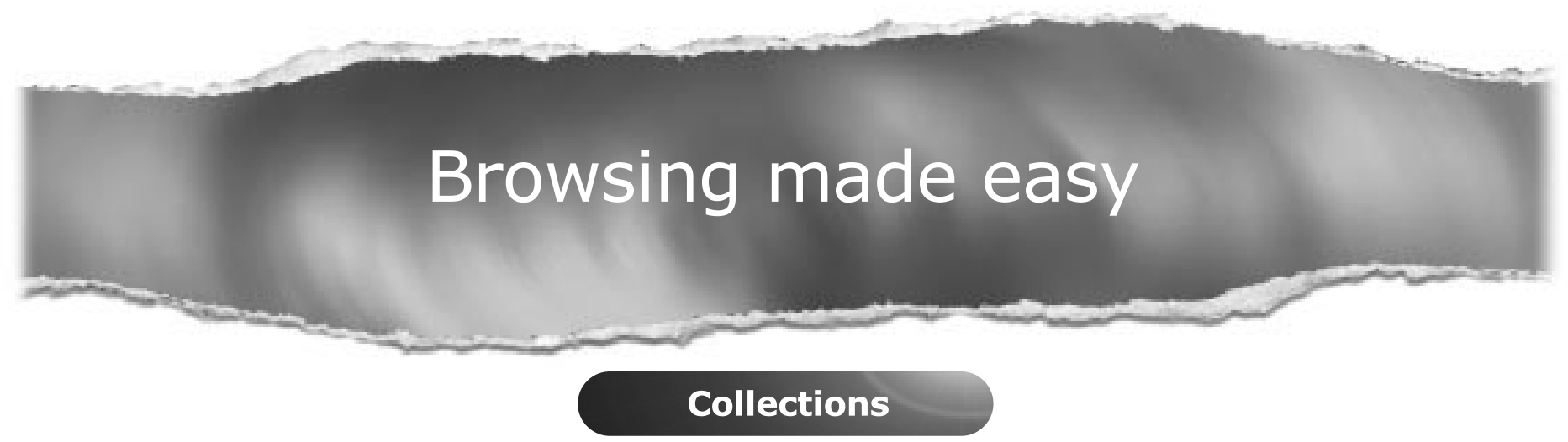

With a single click Collections allows you to find all articles that have been published in your chosen subject. Select from over 200 clinical and non-clinical topic collections and/or cross search other specialist journals, the BMJ and Cochrane Reviews

www.archdischild.com 